\title{
MULTIPLICITY THEORY AND THE OUTER BOUNDARY
}

\author{
JOHN S. SPRAKER
}

(Communicated by Paul S. Muhly)

\begin{abstract}
Let $f$ be a conformal map from the unit disk onto a simplyconnected region $R$. Multiplication by $f$ on $L^{2}$ is a normal operator. In this paper it is shown that the outer boundary of $R$ is a set of multiplicity one for this operator.
\end{abstract}

In what follows, let $R$ be an open bounded simply-connected region in the complex plane $C$. Let $D$ be the open unit disk and $f: D \rightarrow R$ be a conformal map. This paper will deal with the multiplicity theory for the normal operator $M_{f}$ on $L^{2}(\partial D)$ defined by multiplication by $f$. The radial limit function associated with $f$ will be denoted by $f$ as well. This operator is of interest because it is the minimal normal extension of the subnormal operator defined by multiplication by the independent variable on $H^{2}(R)$.

Abrahamse and Kriete have established a connection between the multiplicity function, $m$, and the cardinality of $f^{-1}$. For more on this see [1-3]. In particular for almost all $x$ on the boundary of $R, m(x) \leq \sharp f^{-1}(x)$. If a set on $\partial R$ has two radial preimages at every point in it then intuitively it is accessible from two different "directions" in $R$. The simplest example of such a set is a slit. We will show that the outer boundary (that is, the unbounded component on $C \backslash \bar{R}$ ) is of multiplicity one, a conclusion suggested by the fact that it cannot be approached from two different sides from within $R$.

Theorem. Let $B$ be any component of $C \backslash \bar{R}$. Then $\sharp f^{-1}(x)=1$ for almost every $x$ on the boundary of $B$ with respect to harmonic measure.

Proof. Let $O=\left\{y \in \partial B \mid \sharp f^{-1}(y)>1\right\}$. For every $y \in O$ choose $y_{1}$ and $y_{2}$ in $\partial D$ such that $f\left(y_{1}\right)=f\left(y_{2}\right)=y$ and define $A_{y} \subseteq \bar{D}$ by $A_{y}=\left\{r y_{i} \mid \frac{1}{2} \leq r \leq 1\right.$, $i=1,2\} \cup\left\{\frac{t}{2} y_{2}+(1-t) y_{1} / 2 \mid 0 \leq t \leq 1\right\}$. Then $f\left(A_{y}\right)$ will be a simple Jordan curve which intersects the boundary of $R$ at the point $y$. Also for $z \in O$ with $z \neq y \quad f\left(A_{y}\right) \cap f\left(A_{z}\right)=\varnothing$. For any $y \in O$ since $B$ is connected and disjoint from $f\left(A_{y}\right)$, it lies in one of the two components of the complement of $f\left(A_{y}\right)$. Clearly if two Jordan curves intersect the boundary of $B$ and $B$ lies in the

Received by the editors May 30, 1989.

1980 Mathematics Subject Classification (1985 Revision). Primary 47B38; Secondary 47B15.

Key words and phrases. Multiplicity, multiplication operator. 
bounded components of their complements the Jordan curves must intersect. Thus there is at most one $y \in O$ such that $B$ lies in the bounded component of $C \backslash f\left(A_{y}\right)$.

Let $A=\left\{z \in O \mid B\right.$ lies in the unbounded component of $\left.C \backslash f\left(A_{z}\right)\right\}$. Let $z, y \in A$. Since $B$ lies in the unbounded components of $C \backslash f\left(A_{z}\right)$ and $C \backslash$ $f\left(A_{y}\right)$ and $f\left(A_{y}\right)$ and $f\left(A_{z}\right)$ both intersect the boundary of $B$, neither curve is internal to the other. Thus the bounded components of the sets $C \backslash f\left(A_{z}\right)$ for $z \in A$ form a disjoint collection of open sets. This means that $A$ is countable, and the result follows.

Corollary. The boundary of any component of $C \backslash \bar{R}$ is a set of multiplicity one. Proof. This follows from the theorem and the result of Abrahamse and Kriete.

This result can be easily extended to multiply connected domains by using the theory of Fuchsian groups and fundamental regions.

\section{BIBLIOGRAPHY}

1. M. B. Abrahamse, Multiplication operators, Lecture Notes in Math., vol. 693, SpringerVerlag, Berlin, 1978, pp. 17-36.

2. M. B. Abrahamse and T. L. Kriete, The spectral multiplicity of a multiplication operator, Indiana Univ. Math. J. 22 (1973), 845-857.

3. T. L. Kriete, An elementary approach to the multiplicity theory of multiplication operators, Rocky Mountain J. Math. 16 (1986), 23-33.

Department of Mathematics, Western Kentucky University, Bowling Green, KenTUCKY 42101 